\title{
I. ARTICLES
}

\author{
MARIAN SZCZODROWSKI
}

Ateneum Szkoła Wyższa w Gdańsku

m.szczodrowski6@upcpoczta.pl

\section{Übungen als Grundlage für fremdsprachliche Einkodierungsprozesse}

\author{
Exercises as a Basis for Foreign-language \\ Encoding-processes
}

\begin{abstract}
Various forms of language-activity influence the acquisition of a foreign language. In this article, the author underlines the importance of foreign-language exercises, and their effectiveness in the developing the learner's encoding of decoded foreign-language information structures in the form of constant structure-matrices. The theories of teaching and learning drawn on in the article point to the variety of methods and degrees of foreign-language acquisition, both through cognitive exercises and through the pattern approach. Such exercises develop receptive and productive language-communication skills.
\end{abstract}

KEYWORDS: learning German as a foreign language, language acquisition, classification of foreignlanguage exercises, process of encoding foreign-language information-structures.

Jede menschliche Tätigkeit, bei der man sich bemüht, möglichst große Geschicklichkeit zu erlangen, bedarf immer steten Übens. Einen besonderen Tätigkeitsbereich des Menschen bilden seine sprachlichen Handlungen, die zunächst im Bereich der Sprechsprache und des Weiteren auf dem Gebiet der Schreibsprache ausgeübt, ausgeführt, ständig entwickelt und vervollkommnet werden (können/sollen). Die sprechsprachlichen Handlungen, nämlich die rezeptiven sowie die reproduktiven und produktiven, begleiten 
den Menschen von seiner Kindheit an bis (mit einigen unvorhergesehenen Ausnahmefällen) zum Ende seines Lebens. Im Grunde genommen hängt die rezeptiv-produktive Beherrschung einer Sprache von den interindividuellen und intraindividuellen Sprachkontakten des Menschen mit seinen Kommunikationspartnern sowie von der persönlichen Leistungsfähigkeit und fertigkeit des Gedächtnisses und den Leistungsfunktionen der sprachlichen Rezeptoren und Effektoren während deren Erlernung und Aneignung ab. $\mathrm{Zu}$ den Ersteren gehört sicherlich seine persönliche Begabung, die sich vor allem in sprachlichen sowie in außersprachlichen Erwerbs- und Erlernungsvorgängen äußert. In den interindividuellen Sprachkontakten werden sprachliche Fähigkeiten und Fertigkeiten erlernt und erworben, die es den Kommunikationspartnern ermöglichen, sprachliche Informationssignale aufzunehmen, vollständig zu dekodieren sowie diese in Form von entsprechenden phonetisch-phonematischen bzw. graphisch-graphematischen und semantisch-grammatischen Strukturmatrizen für bestimmte Zeit im Gedächtnis (genauer im Sprachzentrum) aufzubewahren, um sie bei Bedarf rezeptiv oder reproduktiv und produktiv zu gebrauchen. Solche Vorgänge kennzeichnen höchstwahrscheinlich sowohl den Erwerb der Muttersprache als auch die Erlernung jeder Fremdsprache; unterschiedlich sind nur die Bedingungen, unter denen sich diese Sprachprozesse vollziehen, und selbstverständlich die Sprachpartner, mit denen man in direkter Verständigungsverbindung steht.

Fremdsprachliches Üben bedeutet einerseits den kennen gelernten aber noch nicht genügend angeeigneten fremdsprachlichen Stoff in allen LehrLern-Situationen und ebenso unter außerschulischen Bedingungen produktiv beständig zu gebrauchen, und andererseits die bereits gelernten und angeeigneten Sprachstrukturen so zu pflegen, dass sich der Schüler dazu angehalten fühlt, den Zustand seines teilweise schon konstruierten fremdsprachlichen Speicher-Mechanismus nicht nur zu erhalten, sondern dessen entsprechende Sprachstrukturmatrizen so aufzubauen, dass einige automatisiert, die anderen fehlerfrei in entstandenen Kommunikationssituationen benutzt werden können. In beiden Fällen handelt es sich um systematische Tätigkeiten in Bezug auf die Herausbildung fremdsprachlicher Fähigkeiten und Fertigkeiten, um die aufgenommenen Signale effektiv zu dekodieren sowie die dekodierten Informationsstrukturen zu speichern und die für die Kommunikation nötigen Informationsstrukturen abzurufen und einwandfrei kodieren zu können. Durch solche Tätigkeiten und in solchen Vorgängen werden die eingespeicherten Strukturmatrizen dynamisiert, was mit Sicherheit zu besseren rezeptiv-produktiven Leistungen beiträgt. 
Dass das zu lernende und anzueignende sowie das kennen gelernte und auch teilweise bereits angeeignete fremdsprachliche Material eingehend $\mathrm{zu}$ üben und zu verankern ist, braucht nicht näher begründet zu werden. Damit sind folgende Fragen verbunden: erstens die nach der lingualen Wesensart der fremdsprachlichen Übungen und zweitens die nach deren Bedeutung im autonomen glottodidaktischen Bezugssystem, in dem sie einen wesentlichen Teil der aufbereiteten Grundlage zur Weitererlernung und Bewältigung der fremdsprachlichen Strukturmatrizen bilden.

Zum ersten Fragekomplex, der die einzelnen zu übenden Sprachstrukturen umfasst, gehört vor allem das Was des Übungsstoffes, wobei sich der zweite auf das Wie bezieht, worunter die praktischen Ausführungen der Aufgaben verstanden werden. Es geht also um zwei grundsätzliche Fragen, die beim Fremdsprachenlernen gelöst und verwirklicht werden müssen. Die eine kennzeichnet sich durch strukturelle Zusammenhänge der Übungsmaterialien und deren Unterrichtsdarbietung, die andere bleibt schließlich offen und erwartet deren erfolgreiche Lehr-Lern-Realisierung, was wiederum positive Ergebnisse beim Fremdsprachenlehren und -lernen bringen soll. Man muss sich aber dessen bewusst sein, dass sowohl die Aufbereitung als auch die Darbietung des Übungsmaterials sowie seine didaktisch-methodischen Realisierungen in schulischen Unterrichtssituationen unterschiedlich sind und sein können.

Welche Resultate durch Übungen in den Fremdsprachenlernvorgängen von den einzelnen Lernenden erreicht werden können, hängt von der Zahl der ausgeführten schriftlichen beziehungsweise mündlichen Übungsaufgaben sowie von den individuellen Leistungsmöglichkeiten der Lernenden selbst ab. Befriedigende Antworten auf diese Fragen können auf mehrerlei Hinsicht gesucht werden: Zum einen sind die fremdsprachlichen Übungen im Allgemeinen als Bestandteil des (autonomen) Lernprozesses und im Besonderen als kognitives Lernen oder imitatives Lernen zu betrachten. Zum zweiten kennzeichnen sich die fremdsprachlichen Übungen durch ihre typischen Eigenarten, d.h. sie dienen entweder der Festigung oder der Anwendung des fremdsprachlichen Stoffes in verschiedenen Übungs- oder Gesprächsformen. Drittens besteht bei manchen Lernenden die Möglichkeit und zugleich aber auch die Notwendigkeit, dass nur einige - beziehungsweise wenige - synthetische Wortstrukturen und bestimmte Satzstrukturen in Übungen eingeprägt werden müssen, um die oft vorkommenden fremdsprachlichen Mängel zu beseitigen.

Angesichts der oben getroffenen Unterscheidungen und der vorgeführten binären Klassifikationen von fremdsprachlichen Übungen lassen sie sich diese zu folgender Darstellung schematisieren: 


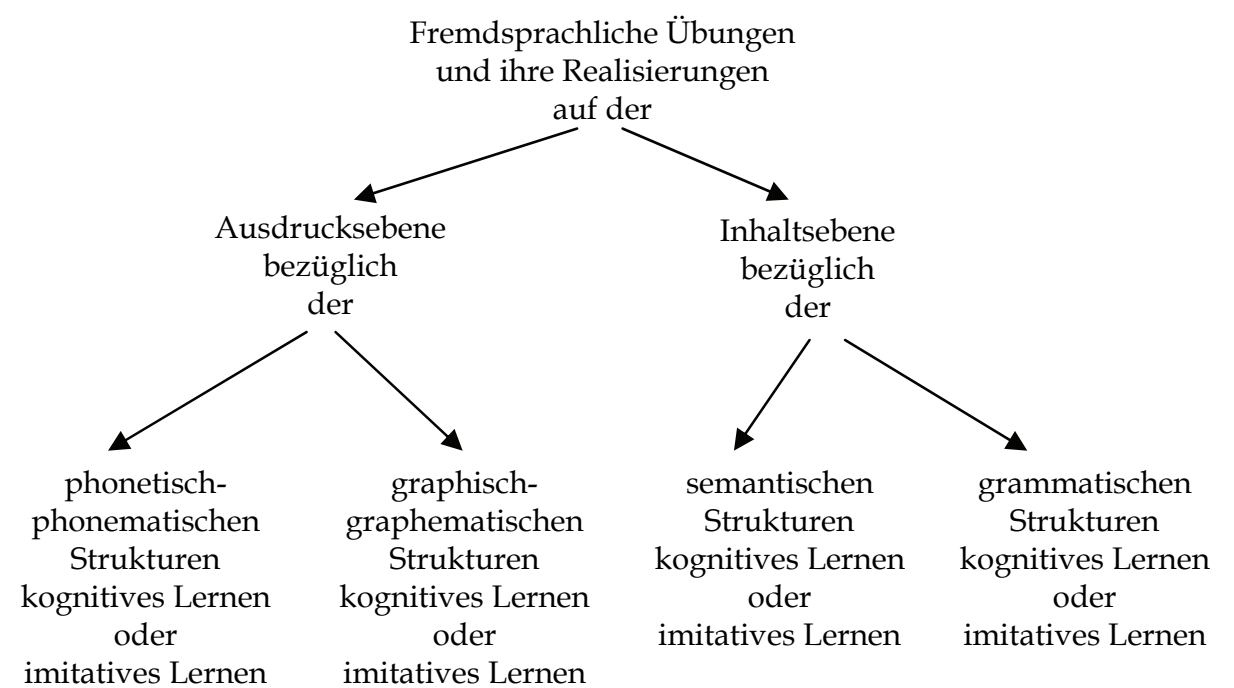

Abb. 1. Binäre Klassifikation der fremdsprachlichen Übungen und deren Realisierungsmöglichkeiten im (autonomen) Lernprozess

Vom wissenschaftstheoretischen und unterrichtspraktischen Standpunkt aus betrachtet sollen sowohl die positiven als auch die negativen Seiten der didaktisch-methodisch angewandten Verfahren bezüglich der Wiederholung und der Festigung des einzuprägenden Stoffes durch die Lernenden auf Grundlage der fremdsprachlichen Übungen und insbesondere der von ihnen erzielten Ergebnisse vom Lehrer analysiert und bewertet werden. Werden die fremdsprachlichen Übungen vom Lehrer selbst vorbereitet, so müssen einerseits ihre Thematik, Art und ausreichende Menge der zu festigenden Strukturen überdacht sowie deren Darbietungsmöglichkeiten in möglicherweise situativen Kontexten und Realisierungsmöglichkeiten in bestimmten Unterrichtseinheiten oder unter außerschulischen Bedingungen vorgeschlagen werden. Darüber hinaus sollen die einzelnen Übungen hinsichtlich der lexikalischen oder grammatischen Strukturen, die für entsprechende Unterrichtseinheit(en) vorgesehen sind, eng mit den im Lehr-LernBuch/Werk vorhandenen Materialien gekoppelt werden. Die vom Lehrer vorbereiteten und in den Unterricht eingeführten Übungen verfolgen das Ziel, entweder den lexikalischen Stoff oder die grammatischen Strukturen in anderen sprachlichen beziehungsweise situativen Kontexten zu erweitern und zu festigen.

Alle Übungen sind als Einkodierungsvorgänge der bereits dekodierten fremdsprachlichen Stoffes zu betrachten, wobei seine einzelnen Strukturen, 
z.B. phonetisch-phonematische bzw. graphisch-graphematische oder semantisch-grammatische, besondere und mehrmalige Einkodierungstätigkeiten verlangen. Anders gesagt: Die gerade genannten Strukturen bilden die Operationsbasis für die Einkodierungsübungen der fremdsprachlichen Strukturen und fußen auf deren Dekodierung und Verarbeitung. Das effektive Lernen und die operationsfähige Aneignung einer Fremdsprache beziehen sich also auf die Einkodierungsvorgänge und auf das Häufigkeitsprinzip des anzueignenden Wortschatzes und der zu bewältigenden Grammatik. Kennzeichnend für die Einkodierungstätigkeiten bezüglich der Sprachstrukturen ist ihr mehrstufiges Einprägen, d.h. sie gelangen zunächst ins Kurzzeitgedächtnis, dann aber ins Langzeitgedächtnis, wo sie in Form von rezeptiv-produktiven Sprech-Sprach-Matrizen und/oder von rezeptiv-produktiven SchreibSprach-Matrizen dauerhaft verankert werden (können) (vgl. dazu Szczodrowski 2009a: 178 ff.; 2009b: 291 ff.). Was die Natur des physischpsychischen Leistungsvermögens des Einzellernenden anbelangt, so ist $\mathrm{zu}$ berücksichtigen, dass die Einkodierung nicht in jedem Lernenden auf gleichartige Weise abläuft. Die jeweiligen Lernfähigkeitsunterschiede betreffen mehrere Bereiche und deren Ebenen, in/auf denen sich die Dekodierungs-, Verarbeitungs-, Einkodierungs-, Speicherungs- und Kodierungsprozesse vollziehen.

\section{FREMDSPRACHLICHE ÜBUNGEN UND IHRE KOGNITIVEN UNTERSTÜTZUNGEN}

Die Erlernung und die Aneignung der Fremdsprache(n) sind immer mit mehreren Unterrichtsformen, unter anderem mit verschiedenartigen Übungen verbunden.

Im kognitiven Lernprozess soll die Bewusstmachung fremdsprachlicher Strukturen den Schülern als wirksame Lernstütze dienen, weil ihnen die Einsicht in das Wesen bestimmter Strukturen und die Erklärung von deren Eigenart bei der Fremdsprachenerlernung behilflich sein kann (vgl. dazu Dakin 1977: 26). Ein reflexives Erfassen des Lernstoffes gewährleistet - wo nur die Notwendigkeit dazu besteht - die Sicherheit des Verständnisses sowohl der $\mathrm{zu}$ übenden phonetisch-phonematischen beziehungsweise der graphisch-graphematischen als auch der semantisch-grammatischen Strukturen und deren bessere Herausbildung und tiefere Einkodierung in Form von fremdsprachlichen Strukturmatrizen mit ihren rezeptiven und (re)produktiven Leistungsfähigkeiten und -fertigkeiten.

Sollen fremdsprachliche Übungen erwartete und befriedigende Lernergebnisse erbringen, dann reicht für deren Realisierungen nicht nur das Prin- 
zip der Wiederholbarkeit aus, weil sie bei einigen Lernenden mehrmaliges mechanisches Repetieren und zugleich viel Übungszeit verlangen, was unter schulisch-fremdsprachlichen Bedingungen kaum möglich ist. In einem solchen Fall wäre es vielleicht angebracht, eine kurze Erläuterung der in den Übungen vorkommenden fremdsprachlichen Gesetzmäßigkeiten zu geben, weil das Üben sowohl häufiger Wiederholungen als auch kognitivsprachlicher Auslegungsprozesse bedarf. Auf diese Weise können die geübten Strukturen tiefer und tauglicher einkodiert werden, wodurch ihre rezeptiven und produktiven Leistungen verbessert und gesteigert werden (können). Solche Prozesse betreffen die Komplexität der Übungsmaterialien, d.h. sowohl ihre Strukturen auf der Ausdrucksebene als auch die auf der Inhaltsebene.

Was die Wiederholbarkeit der fremdsprachlichen Übungen anbelangt, so liegt ihr Wesen darin, dass sie die zu übenden Strukturen berücksichtigt, die in möglichst verschiedenen Kommunikationssituationen sowie in dialogischen und ebenso in polylogischen Interaktionen durchgeführt werden können.

Dass die Übungsabläufe nicht allein auf die sprachlichen Strukturen zu beschränken sind, sondern in einem Abhängigkeitsverhältnis zum Inhalt, genauer gesagt zu bestimmten Themen stehen, hat Krüger (1985: 17 ff.) stark betont. Er weist auf die Kommunikationsfertigkeiten hin, denen die Verstehens- und Mitteilungsfähigkeiten zugrunde liegen und unterstreicht, dass die für die Kommunikation benötigten fremdsprachlichen Fähigkeiten und Fertigkeiten in den Übungsabläufen tiefer herausgebildet werden sollen. Eine durchdachte Verbindung der (rein) fremdsprachlichen Strukturen mit ihrem kommunikativen Zusammenhang bildet seines Erachtens eine Vorstufe zur kommunikativen Anwendung der zu lernenden und der gelernten Strukturen. Für die angebotenen Übungsabläufe werden vier Phasen erstellt, deren Ziel es ist, sich die Fertigkeiten nicht separat einzuprägen, sondern sie in verschiedenen syntagmatisch-syntaktischen Konstruktionen möglich miteinander zu verknüpfen und zu integrieren. In den Phasen, in denen die $\mathrm{zu}$ lernenden Informationen dargeboten werden, sollen sie in vier aufeinanderfolgenden Schritten aufgenommen und verarbeiten werden (vgl. Krüger 1985 20-25).

Entwicklung von Verstehensleistungen

Der neu präsentierte Text soll im Fremdsprachenunterricht von den Lernenden verstanden werden, wobei der Verstehensgrad durch den Lehrer überprüft wird und der ebenso bei komplizierteren Texten behilflich ist. Kennzeichnend für die Übungen in dieser Phase ist ihr mechanisch- 
reproduktiver Charakter; zu ihnen gehören beispielsweise Richtig-Falsch-, Verbindungs- und Antwort-Auswahl-Übungen.

Schaffung von Grundlagen der Verständigungsfähigkeit

Nach dem Verstehen der entschlüsselten Informationen werden die dem Inhalt des dargebotenen Textes entsprechen Strukturen unter besonderer Berücksichtigung der grammatisch-syntaktischen Konstruktionen gewählt und reproduktiv geübt. Es sind vor allem Einsetzübungen, Spiele zur Aktivierung von Wortschatz und Grammatik usw., welche einer starken Steuerung bedürfen und allmählich die freie Anwendung des geübten Stoffes vorbereiten.

Entwicklung von Verständigungsfähigkeit

Typisch für diesen Schritt sind das Üben und der Gebrauch bestimmter Techniken, und zwar Textentschlüsselung und Notizenmachen, aber auch gelenkte Äußerungen und Texterzeugung nach vorgegebenen Stichwörtern.

Freie Meinungsäußerung/Anwendung in Simulationen

Fremdsprachliche Handlungen basieren in dieser Phase auf der Entwicklung und der Aneignung von Verstehens- und Mitteilungsleistungen, die es den Lernenden ermöglichen, die erworbenen Kenntnisse, die sozialinteraktiven Verhaltensweisen und das fremdsprachliche Können ,spontan' in den Kommunikationssituationen anzuwenden. Gemeint ist hier insbesondere das fremdsprachliche Handeln außerhalb des institutionalisierten Unterrichts.

\section{FREMDSPRACHLICHE ÜBUNGEN UND IHRE MECHANISCHEN AUSFÜHRUNGEN}

Mechanische Ausführungen der fremdsprachlichen Strukturen basieren auf dem Grundsatz des imitativen Lernverfahrens, bei dem sie durch mehrmalige Wiederholung ins Gedächtnis eingeprägt werden. Kennzeichnend für die mechanischen Wiederholungsübungen sind vor allem mündlich-synthetische Nachahmungen, die ausgewählte Wortstrukturen in syntagmatisch-syntaktischen Konstruktionen beinhalten und in denen die richtigen Wortendungen und deren korrekte Verbindungen im Satz, aber auch schriftlich-synthetische Aufgaben nach entsprechenden Satzbaumustern, in denen bestimmte Strukturen auf didaktisch-methodische Weise hervorgehoben und phonetisch-phonematisch bzw. graphisch-graphematisch eingeschliffen werden, Berücksichtigung finden. Für die Schüler, denen das kognitive Fremdsprachenlernen Schwierigkeiten bereitet, sind synthetische Nachahmungen der phonetisch-phonematischen Strukturen bzw. die Darle- 
gung und Erklärung der graphisch-graphematischen Regeln oder die Analogiebildungen im Bereich der syntagmatischen und syntaktischen Konstruktionen als nutzbringendes und effektives Lernverfahren zu verstehen. Durch die Einübung der syntaktischen Konstruktionen werden ihre einzelnen Strukturen verfestigt, was des Weiteren zur erfolgreicheren Aneignung von Sprachgewohnheiten beiträgt sowie beim situationsbezogenen Gebrauch behilflich sein kann.

Betrachtet man den Prozessverlauf der Struktureneinprägung unter dem areflexiven Aspekt, so lässt sich feststellen, dass durch solche Übungstätigkeiten drei Ziele erreichen werden können:

1. Die Festigung des fremdsprachlichen Stoffes verursacht entsprechendes Sprachverhalten;

2. Daraus resultiert auch das richtige Sprachverhalten in entstandenen bzw. in zu entstehenden Kommunikationssituationen;

3. In der Teilnahme an den Kommunikationssituationen werden zugleich die Sprechfähigkeiten und -fertigkeiten des Fremdsprachenlernenden immer weiterentwickelt sowie tiefer und tauglicher einkodiert.

Wie das Einüben der morphostrukturellen Eigenschaften beispielsweise von deutschen Tempusformen Perfekt und Präteritum stattfinden kann, hat Latzel (1982: passim) im seinem Zusatzmaterial gut konzipiert und auch teilweise in Verbindung mit bildlich dargestellten Situationen bearbeitet. Mit Recht stellt er fest, dass die semantisch-pragmatischen Unterschiede zwischen den bereits erwähnten Tempora als komplizierte Didaktikprobleme für diejenigen anzusehen sind, die Deutsch als Fremdsprache lernen. Sein Übungsbuch ist auch für Anfänger (s. Übungsteil A und B, S. 7-60) geeignet, aber vor allem für fortgeschrittene Deutschlernende gedacht, die ihre Hörer/ Sprecher-Fähigkeiten/Fertigkeiten ausbauen, verbessern und bereichern wollen.

Ein gutes und wertvolles Lehr-Lern-Werk, das bestimmte und komplizierte Problembereiche der deutschen Grammatik umfasst, haben Karin Hall und Barbara Scheiner konzipiert und herausgegeben (1999). Ihre Übungsgrammatik Deutsch als Fremdsprache für Fortgeschrittene kann als grundlegende Sprachquelle für die Lernenden, die das Deutsche sowohl in der Gruppenarbeit als auch im autonomen Unterricht besser beherrschen wollen, betrachtet werden. Die in diesem Werk erarbeiteten und in Übungen dargestellten grammatischen Fragen sollten gründlich behandelt werden, um sie als ausgewählte und angeordnete Strukturen in syntagmatischsyntaktischen Konstruktionen zu erweitern, zu vertiefen und $\mathrm{zu}$ festigen. Der Übungsgrammatik liegen zwei wesentliche Grundsätze zugrunde, und zwar werden 1. die grammatischen Konstruktionen mit zahlreichen Beispie- 
len erklärt, um sie in Einzelschritten und dann in Gesamtübungen einzuprägen, 2. sind die komplizierten grammatischen Konstruktionen in bestimmte sprachliche Kontexte eingebaut, wodurch ihre Strukturen mit entsprechender Thematik behandelt werden können. Solche erreichbare Zielvorhaben haben die Autorinnen im Vorwort des Lehr-Lern-Werkes formuliert und konsequent ausgeführt (S. 3 und passim). Dass die zu übenden grammatischen Ordnungsprinzipien nicht nur in Sätzen, sondern auch in kombinierter Weise in zusammenhängenden Texten vorkommen, ermöglicht und garantiert, komplex(er)e Sprachstrukturen zu durchschauen und diese auch produktiv in entstandenen Kommunikationssituationen zu gebrauchen.

Bewertet man die Grundprinzipien der fremdsprachlichen Übungen und ihre rezeptiv-produktiven Konsequenzen, so sollte unbedingt auf die Verschiedenartigkeit der Übungsformen hingewiesen werden, die jeweils auf jene Fähigkeiten und Fertigkeiten abzielen, die bezüglich bestimmter Sprachstrukturen in den Lernenden herausgebildet werden sollen und können. Eine breite Skala von Übungen findet der Interessente im Werk Didaktik des Fremdsprachenunterrichts -Deutsch als Fremdsprache, das vom einem Autorenkollektiv unter Leitung von Günter Desselmann und Harald Hellmich (1981: 75 ff.) herausgegeben wurde. Die Zielkonzeption der Autoren bestand darin, möglichst zahlreiche Übungen, beispielsweise mündliche und schriftliche, präkommunikative, rezeptive und produktive, aber auch Differenzierungs-, Erfassungs-, Nachahmungs-, Nachsprech-, Substitutions-, Transformations-, Wortschatzübungen u.v.a.m., zusammenfassend darzustellen und zu zeigen, wie die Übungsmaterialien auf verschiedenen Lehr- und Lernstufen gestaltet, dargeboten und realisiert werden und welche Ergebnisse die Lernenden im Bereich der Herausbildung und der Entwicklung des fremdsprachenkommunikativen Könnens erreichen.

Praktische und konstruktive Vorschläge zur Lösung didaktischmethodischer Probleme, und zwar welche deutsche Wendungen, die im Fremdsprachenunterricht in Festigungsübungen rezeptiv, reproduktiv und produktiv beherrscht werden sollen, legt Strauss (1988: 36-41) vor. Seine Hinweise und Empfehlungen beziehen sich nicht nur stricte auf die Festigungsübungen, sondern auch auf den ganzen Lernweg, auf dem der Übungsvorgang in drei Lernschritten realisiert wird, nämlich in der Aufnahme-, Festigungs- und Anwendungsphase. Die bereits dargestellte Lernwegaufteilung stimmt - vom didaktisch-methodischen Standpunkt aus betrachtet - mit den Termini der Einführungs-, Übungs- und Transferphase überein. Jedem Lernschritt liegen folgende Teillernziele zugrunde. Es sind folgende: 
- Fähigkeit zum allgemeinen Textverständnis, aber insbesondere zum Verständnis der Bedeutungen der anzueignenden Wendungen;

- Fähigkeit zur ersten (noch nicht flüssig-fehlerfreien) Reproduktion der zu erlernenden Wendungen;

- Fähigkeit zur flüssigen und spontanen Reproduktion der zu (re-)aktivierenden (= zu dynamisierenden) Wendungen.

In den drei aufeinanderfolgenden Phasen hat Strauss die konkreten Lerneraktivitäten, Lernziel(e) angeordnet und ebenso die Lern- und Lehrtechniken akribisch herausgearbeitet. Die Lerneraktivitäten basieren auf einem Ausgangstext, der bestimmten Lernzielen angepasst ist und den man auf Grund der vorgebrachten Lern- bzw. Lehrtechniken, die sich beispielsweise auf Motivations-, Textdarbietungs-, Verbesserungsstrategien des Grobverständnisses (Aufnahmestufe), oder das Überwiegen von Festigungsübungen mit gehäufter Wiederholung von Wendungen (Festigungsstufe), aber auch auf das Überwiegen von Anwendungsübungen mit Wiederholung von mehreren neuen und bereits gefestigten Wendungen (Anwendungsstufe) beziehen, ergänzen muss.

\section{ALLGEMEINE SCHLUSSFOLGERUNGEN}

Die oben dargestellten zwei grundlegenden Arten der fremdsprachlichen Übungen - und wahrscheinlich nicht nur solche - kennzeichnen sich durch folgende Eigenschaften: einerseits sind sie geeignete Maßnahmen zur Verhütung von verschiedenartigen Fehlern, die durch die Lernenden im Unterricht gemacht werden (können). Andererseits haben sie die sinnvollen Aufgaben, die in produzierten Äußerungen bzw. Texten auftretenden fremdsprachlichen Unrichtigkeiten aufzudecken, zu verbessern und die sprachlichen Einheiten fehlerfrei so zu festigen bis ihre Korrektheit erreicht ist und vom Lehrenden akzeptiert wird.

Fremdsprachliche Übungen umfassen bestimmte ausgewählte Strukturen, die vor allem im Rahmen syntagmatisch-syntaktischer Konstruktionen Umsetzung finden. Aus den syntagmatisch-syntaktischen Konstruktionen können nur spezielle Strukturen, beispielsweise entweder aus dem Ausdrucksbereich oder aus dem Inhaltsbereich, angeordnet und für das Einüben aufbereitet werden. Es ist jedoch möglich, dass die in den syntagmatisch-syntaktischen Konstruktionen vorkommenden Strukturen in größere Satzgefüge eingebaut und geübt werden. In solch einem Fall bemüht man sich, all diese Strukturen allmählich zu bewältigen. Im Falle von derart umfassenden Übungen hat man es mit einer doppelten Aufgabe zu tun: Zum 
einen sollten die syntagmatisch-syntaktischen Strukturen tiefer verankert, zum anderen so eingeschliffen werden, dass sie zu Sprachgewohnheiten werden.

Die tiefe Verankerung der Strukturen versteht sich als deren Festigung hinsichtlich der semantisch-grammatischen Strukturmatrizen und das Einschleifen bedeutet, dass sie ihre automatische Geläufigkeit bezüglich der phonetisch-phonematischen bzw. ihre Richtigkeit in Hinblick auf die graphisch-graphematischen Regeln erreichen. Die Verbindung jener doppelten Aufgabe wirkt letzten Endes als Lernverstärkung bezüglich der geübten Strukturen und führt zu ihrer rezeptiv-produktiven und operationsfähigen Aneignung. $\mathrm{Zu}$ charakteristischen Eigenarten der Doppelaufgabe gehören sowohl sprachlich-bewusste Tätigkeiten, welche die Aufmerksamkeit des Lernenden auf die Bedeutungselemente der fremdsprachlichen Strukturen lenken, wodurch wiederum der Lernprozess kognitiv gesteuert wird, als auch die Gewöhnungsübungen, die zur automatischen Beherrschung der Sprachgewohnheiten führen.

Beide Lerntätigkeiten, d.h. das kognitive und das imitative Üben, die sich in glottodidaktischen Konzeptionen bekanntlich prinzipiell voneinander unterscheiden, haben ein gemeinsames Lernziel, das darin besteht, fremdsprachliche Mittel in Form von dargebotenen Strukturkonstruktionen so zu bewältigen, dass man mit ihnen in entstandenen Kommunikationssituationen möglichst einwandfrei operieren kann. Wenn solche rezeptivproduktiven fremdsprachlichen Handlungsfähigkeiten und -fertigkeiten bezüglich des Übungsstoffes herausgebildet sind, die es dem Lernenden erlauben, als Empfänger und Sender an der Kommunikation teilzunehmen, dann kann angenommen werden, dass das im Unterricht zu verwirklichende übergeordnete Lernzielvorhaben erreicht worden ist. Für den weiteren Fremdsprachenunterricht bedeutet dies auch, dass man sich auf Grund des angeeigneten Lernstoffes mit anderen Sprachproblemen beschäftigen oder dem Unterrichtsprogramm gemäß - schon zu neuem Lehr-Lern-Stoff übergehen kann.

Wie in jeglicher Lehrtätigkeit so auch in jeder Form der fremdsprachlichen Übungen sollen/müssen die glottodidaktisch-methodischen Tätigkeiten des Lehrenden bezüglich der Aufnahme und der ganzheitlichen Dekodierung der übertragenden Informationsstrukturen durch den Lernenden zielführend gesteuert und auf Grund seiner reproduktiven und produktiven Informationskodierung kontrolliert werden. Dabei ist ebenso zu betonen, dass der Fremdsprachenlerner als Kommunikationsteilnehmer, d.h. als Empfänger oder als Sender bestimmten autonomen Steuerungsvorgängen 
unterliegt und - wenn nur die Möglichkeit besteht - auch sich selbst kontrolliert oder kontrollieren kann.

Da die fremdsprachlichen Übungen stets in hohem Maße durch den Lehrenden wie auch durch die Lernenden, insbesondere im Selbstunterricht, gelenkt und kontrolliert werden, bedürfen sie systematischer Beobachtungen und Erfolgsbewertungen. Sollen die Übungen zur dauerhafteren Einkodierung des kennen gelernten Materials bei jedem Lernenden beitragen, so beinhaltet ihr Ziel mehr als nur das Einprägen bestimmter Sprachstrukturen. Das Ziel besteht auch darin, dass das Übungsmaterial an solche Kommunikationssituationen angepasst wird, die von einfachen, beispielweise aus Frage und Beantwortung bestehenden, Unterrichtshandlungen ausgehen und schrittweise zu dialogischen und polylogischen Gesprächen oder zu einer monologisch verkürzten Darstellung ausgewählter Themen ausgearbeitet werden. Kennzeichnend für die fremdsprachlichen Unterrichtssituationen sind also solche Sprechhandlungen, die den Lernenden die Möglichkeit einräumen, das Gelernte und das Geübte in neue Kommunikationssituationen zu übertragen und somit produktiv anzuwenden. Bei der Strukturierung der fremdsprachlichen Übungen, ungeachtet dessen, welche Grammatik- oder Wortschatzprobleme ihnen zu Grunde liegen, richten sich die Autoren immer nach den gestellten Zielen (mehr dazu Neuner 1985: 7 ff.).

Es bleiben schließlich einige grundsätzliche Fragen offen, und zwar:

1. Auf welche Weise wird der einzuübende fremdsprachliche Lernstoff in entsprechenden Satzstrukturen zusammengefasst, angeordnet, didaktisch-methodisch dargeboten und empfangen;

2. Welche und wie viele Übungen sowie in welchen Kommunikationsbzw. Unterrichtsformen, und zwar im Frontal-, Gruppen- (Großgruppenoder Kleingruppen-), Partner- oder Selbstunterricht sollen sie durchgeführt werden;

3. Nimmt der Lehrende als Beobachter und Kontrolleur an den Übungen teil, so taucht das noch nicht didaktisch gelöste Problem auf, ob er manche Lernenden mit gezielten Aufgaben aktivieren soll/muss, damit sie nicht nur rezeptiv, sondern auch produktiv in die fremdsprachlichen Interaktionen eingreifen.

Der Sinn der fremdsprachlichen Übungen, die sowohl unter kognitiver Mitwirkung als auch auf Grund mechanischer Ausführungen realisiert werden, liegt vor allem in der festen Einkodierung von Wörtern, Wortgruppen und Satzstrukturen, die dann entweder umgeformt oder syntagmatischsyntaktisch ausgebaut werden können. Alle Übungen tragen zur besseren Fremdsprachenerlernung bei und bieten ebenso die Gelegenheit, die neu 
konstruierten Wort- oder Satzstrukturen in anderen Kommunikationssituationen anzuwenden.

$\mathrm{Zu}$ den oft diskutierten Problemen, mit denen sich die Vertreter der theoretischen Konzepte des Fremdsprachenerlernung beschäftigten und noch immer befassen, gehören ebenso die Zusammenhänge zwischen Bewusstsein und Automatisierung im Fremdsprachenunterricht (vgl. dazu Helbig 1981: 67 ff.). Diese Fragen betreffen im Allgemeinen den Fremdsprachenunterricht und im Besonderen die fremdsprachlichen Übungen, die als wesentliche Komponenten der Lernvorgänge $\mathrm{zu}$ betrachten sind. $\mathrm{Zu}$ heftigen Auseinandersetzungen kam und kommt es jeweils dann, wenn man akzeptiert, dass nur das Bewusstsein, d.h. das Wissen um die Strukturen der sprachlichen Systeme und deren Erlernung/Beherrschung für Kommunikationszwecke, oder lediglich die Automatisierung, die auf dem spontan ablaufenden Rezeptionsvorgang, der nicht von Bewusstsein beeinflusst wird, beruht, den Erlernungs- und Aneignungsprozessen der Fremdsprache unter schulischen Bedingungen zugrunde liegt. Eine solche ausschließliche Anerkennung und Annahme des Lernverfahrens verflacht das Wesen des Lernprozesses, das angesichts seiner Komplexität nicht einseitig, sondern unter mehreren Aspekten analysiert und realisiert werden sollte. Also veranlassen und berechtigen die Komplexität des Lernprozesses einerseits und die individuellen Eigenschaften des Einzellerners andererseits zur Akzeptierung solcher lerntheoretischen Konzepte, die sowohl das Bewusstsein als auch die Automatisierung (Imitation) berücksichtigen, weil sie sich nicht gegenseitig ausschließen, sondern - so Helbig 1981: 67 und 69 - sich wechselseitig bedingen und in einem integrativen Verhältnis zueinander stehen. Beim Fremdsprachenunterricht wird nicht nur dem Wissen, d.h. den benötigten Kenntnissen von zu lernenden und gelernten Fremdsprachenstrukturen, sondern auch dem fremdsprachlichen Können, nämlich den zu erwerbenden bzw. zu erworbenen Fähigkeiten und Fertigkeiten, die sich in rezeptiven und produktiven Leistungen realisieren, erhebliches Gewicht beigemessen.

Diese Konstatierung stimmt mit der Meinung überein, dass die zur Zeit dominierende moderne Methode keinesfalls als glottodidaktisches Allheilmittel anzusehen ist.

Wie verschiedenartig konstruierte Übungen mit Handlungen und Aktivitäten, mit Sprache und Sprechabsichten in vielen dargestellten Kommunikationssituationen, die sowohl mit einzelnen Wörtern oder Syntagmen als auch mit Sätzen ergänzt werden sollen, aussehen können, zeigt das Übungsbuch von Jutta Müller und Heiko Bock (1995), in dem der deutsche Grundwortschatz, bereichert mit visuellen Mitteln, zu trainieren und anzueignen ist. 
Eine kurz aber klar gefasste Darstellung über die linguistisch und didaktisch-methodisch ausgearbeiteten und vorgeschlagenen Konzepte bezüglich des Fremdsprachenunterrichts gibt Szulc (1976: 57-76), wenn er auf die Theorien des amerikanischen Strukturalismus, der audio-lingualen HabitMethode, der Pattern-Practice Methode, der behavioristischen Verhaltenstheorie, des kognitiven Lernens, N. Chomskys Spracherwerbstheorie u.v.a. eingeht und ihre wesentlichen wissenschaftlich begründeten Aspekte zusammenstellt und auch einigermaßen kritisch und sachlich beurteilt. Mit diesem Überblick werden darüber hinaus folgende Ziele verfolgt: Eine Informierung über die Forschungsrichtungen und -perspektiven, die ein Optimieren des Fremdsprachenunterrichts ermöglichen, und das Hinweisen auf neue theoretische Konzepte, welche die Effektivität des Lernprozesses einer Fremdsprache erhöhen (sollen) und deren Anwendung in der Kommunikation erleichtern werden.

Bei genauerem Hinsehen zeigt sich aber, dass in den zahlreichen Theorien immerfort die Fragen der habituellen Automatisierung und des bewussten Lernens im Fremdsprachenunterricht auftauchen. $\mathrm{Zu}$ bemerken sei noch Folgendes: In jeder neuen lerntheoretischen Betrachtungsweise werden einerseits ihre (starken) Pro-Argumente für andere (bessere und effektivere) praktische Lösungen vorgeschlagen und hervorgehoben, andererseits werden ebenso die Contra-Argumente gegen die bisherigen Konzepte vorgebracht.

\section{LITERATURVERZEICHNIS}

Dakin, J.,1977. Vom Drill zum freien Sprechen. Übungsformen für Sprachlabor und Klassenraum. Freudenstein, R. (Hrsg. u. Übers.). München: Langenscheidt-Longman.

Didaktik des Fremdsprachenunterrichts. (Deutsch als Fremdsprache), 1981. Von einem Autorenkollektiv unter Leitung von Desselmann, G. und Hellmich, H. Leipzig: VEB Verlag Enzyklopädie.

Hall, K., Scheiner, B., 1999. Übungsgrammatik Deutsch als Fremdsprache für Fortgeschrittene. Ismaning: Verlag für Deutsch.

Helbig, G., 1981. Sprachwissenschaft - Konfrontation - Fremdsprachenunterricht. Zur Theorie und Praxis des Deutschunterrichts für Ausländer. Leipzig: VEB Verlag Enzyklopädie.

Krüger, M., 1985. Übungsabläufe im Fremdsprachenunterricht. In: Neuner G., Krüger M., Grewer U. (Hrsg.). Übungstypologie zum kommunikativen Deutschunterricht. Berlin et al.: Langenscheidt, 17-28.

Latzel, S., 1982. Übungen zum Gebrauch von Perfekt und Präteritum im Deutschen. Exercises for the Use of the Perfect and Imperfect Tenses in German. München: Max Hueber Verlag.

Müller, J., Bock, H., 1995. Grundwortschatz Deutsch, Übungsbuch. Berlin et al.: Langenscheidt. 
Neuner, G., 1985. Zum Wandel der Prinzipien und Übungsformen in der Fremdsprachenmethodik. In: Übungstypologie zum kommunikativen Deutschunterricht. In: Neuner G., Krüger M., Grewer U., (Hrsg.). Berlin et al.: Langenscheidt, 7-12.

Strauss, D., 1988. Didaktik und Methodik Deutsch als Fremdsprache. Eine Einführung. Berlin et al.: Langenscheidt.

Szczodrowski, M., 2009a. Einkodierung von fremdsprachlichen Strukturen als grundlegender Lern- und Aneignungsprozess. In: Białek E., Rzeszotnik J., Tomiczek E. (Hrsg.). Auf der Suche nach Humanitas. Festschrift für Prof. Dr. Irena ŚwiattowskaPrędota. Dresden: Oficyna Wydawnicza ATUT, Wrocławskie Wydawnictwo Oświatowe. Wrocław: Neisse Verlag, 177-183.

Szczodrowski, M., 2009b. Interiorisation und Exteriorisation als Glottokode-Prozesse. In: Kolago L. (Hrsg.). Studia Niemcoznawcze / Studien Zur Deutschkunde, Band XLII, 289295.

Szulc, A., 1976. Die Fremdsprachendidaktik. Konzeptionen - Methoden - Theorien. Warszawa: PWN. 
